\title{
Atomic Force Microscopic Evidence for Z-band as a Rigid Disc Fixing the Sarcomere Structure of Skeletal Muscle
}

\author{
Jun'ichi Wakayama, Yoshihiro Yoshikawa, Toshihiro Yasuike, and Takenori Yamada* \\ Department of Physics (Biophysics Section), Faculty of Science, Science University of Tokyo, Shinjuku-ku, \\ Tokyo 162-8601, Japan
}

\begin{abstract}
Atomic force microscopic images of single skeletal myofibrils showed periodical broad filamentous bands interspaced with narrow rigid bands corresponding to the sarcomere structures of skeletal muscle (Yoshikawa, Y., Yasuike, T., Yagi, A., and Yamada, T. 1999. Biochem. Biophys. Res. Comm., 256: 13-19). In order to identify the narrow rigid bands, comparative studies were made for intact single myofibrils and those treated with calcium-activated neutral protease by use of atomic force microscopy. It was found that (a) the periodical narrow rigid bands present in intact myofibrils were completely absent in myofibrils treated with calcium-activated neutral protease, and that (b) myofibrils treated with calcium-activated neutral protease were very fragile compared with intact myofibrils. As calcium-activated neutral protease selectively removes Z-bands of myofibrils (Reddy, M. K., Etlinger, J. D., Rabinowitz, M., Fischman, D. A., and Zak, R. 1975. J. Biol. Chem., 250: 4278-4284), these results clearly indicate that (a) the narrow rigid bands are the $Z$-bands, and that (b) the $Z$-bands are the essential disc supporting the sarcomere structure of skeletal muscle.
\end{abstract}

Key words: skeletal muscle/Z-band/M-line/sarcomere/calcium-activated neutral protease (CANP)/atomic force microscopy (AFM)

Skeletal muscle is composed of a linear array of sarcomeres interspaced with Z-bands (Bagshaw, 1993). At the center of sarcomere exists the M-line. The Z-bands are zigzag networks composed of $\alpha$-actinin that anchor the tip of thin filaments, while the M-line is composed of M-proteins that bundle together the middle of thick filaments (Squire, 1981). The contractile force, produced by the interaction between thin and thick filaments, is transmitted to the Z-bands via the thin filaments. It is therefore essential that the myofilament lattice structure in each sarcomere is kept intact in contracting muscle fibers to stably produce force. Based on the above structure of the sarcomere, it is generally believed that both the Z-bands and the M-lines are the essential components supporting the myofilament lattice of skeletal muscle (Squire, 1981).

Recently we investigated single myofibrils of skeletal muscle by use of atomic force microscopy (AFM), and ob-

\footnotetext{
* To whom correspondence should be addressed: Department of Physics (Biophysics Section), Faculty of Science, Science University of Tokyo, Tokyo 162-8601, Japan.

Tel: $+81-3-3260-4271$ ext. $2415, \quad$ Fax: $+81-3-5261-1023$

E-mail: yamada@rs.kagu.sut.ac.jp

Abbreviations: AFM, atomic force microscope; CANP, Ca-activated neutral protease.
}

tained images showing broad filamentous bands interspaced with narrow rigid bands (Yoshikawa et al., 1999). Based on the comparison between AFM images and optical microscopic images of myofibril preparations, we have tentatively assigned the narrow rigid band as the Z-band and the broad band as the A-band, but could not determine the Mlines in the AFM images. It is known, however, that the AFM imaging is subject to artifacts produced by scanning the tip of AFM cantilever to capture images, which mechanically deforms the elastic surface of biological preparations (Engel and Gaub, 1998). As both the Z-bands and the Mlines have the same periodicity along myofibrils (Bagshaw, 1993) and are thought to be rigid structures, it may be argued that the narrow rigid band observed in AFM images of myofibrils could be the M-line or some component other than the Z-band. To clarify this, we made comparative AFM studies for intact myofibrils and those treated with calciumactivated neutral protease (CANP) since CANP is known to selectively remove the Z-bands of muscle fibers (Reddy et al., 1975). 


\section{Materials and Methods}

\section{Sample preparation}

Myofibrils were prepared by homogenizing glycerinated muscle fibers of rabbit psoas muscle with a Polytron homogenizer (PT1200; Kinematica, Switzerland) as detailed previously (Yuri et al., 1998). Myofibrils thus prepared were stored on ice, and used for experiments within one hour.

A drop of myofibril suspension in $155 \mathrm{mM} \mathrm{K}^{+}$-propionate, 5 $\mathrm{mM} \mathrm{MgCl}_{2}, 5 \mathrm{mM}$ ATP, and $20 \mathrm{mM}$ imidazole, $\mathrm{pH}$ 7.0, was put on the surface of a clean cover slip. It was allowed to stand until myofibrils attached to the surface of the cover slip. After washing out unattached myofibrils with a solution of $155 \mathrm{mM} \mathrm{K}^{+}$-propionate, 5 $\mathrm{mM} \mathrm{MgCl} 2,10 \mathrm{mM}$ EGTA, and $20 \mathrm{mM}$ imidazole, $\mathrm{pH} 7.0$, the cover slip was adhered to the bottom of a culture dish. The culture dish was then filled with a bathing solution $\left(155 \mathrm{mM} \mathrm{K}^{+}\right.$-propionate, $5 \mathrm{mM} \mathrm{MgCl} 2,10 \mathrm{mM}$ EGTA, and $20 \mathrm{mM}$ imidazole, $\mathrm{pH}$ 7.0). Myofibrils attached to the cover slip adhered to the bottom of the culture dish thus prepared were used for experiments of intact myofibrils.

CANP-treatments of myofibrils were made as follows. After myofibrils were attached to cover slip adhered to the bottom of a culture dish as described above, they were treated with CANP by replacing the bathing solution in the culture dish with a CANP solution containing $0.15 \mathrm{mg} / \mathrm{ml} \mathrm{CANP}$ in $50 \mathrm{mM} \mathrm{K}^{+}$-propionate, 10 $\mathrm{mM} \mathrm{CaCl}_{2}$, and $20 \mathrm{mM}$ imidazole, $\mathrm{pH}$ 7.0. After standing for 10 $20 \mathrm{~min}$ at room temperature, during which time the disappearance of Z-bands from myofibril preparations was observed under optical microscope, the reaction was stopped appropriately by washing out the CANP solution with the bathing solution $\left(155 \mathrm{mM} \mathrm{K}^{+}\right.$-propionate, $5 \mathrm{mM} \mathrm{MgCl} 2,10 \mathrm{mM}$ EGTA, and $20 \mathrm{mM}$ imidazole, $\mathrm{pH}$ 7.0). Myofibrils attached to cover slip adhered to the bottom of the culture dish thus prepared was used for experiments of CANPtreated myofibrils.

\section{AFM experiments}

An AFM incorporated into an inverted optical microscope (NV2500; Olympus Optical Co., Tokyo, Japan) was used (Yoshikawa et al., 1999). Commercially available cantilevers made of silicon nitride (spring constant, $0.02 \mathrm{~N} / \mathrm{m}$; tip radius, $50 \mathrm{~nm}$ ) were used.

AFM experiments of myofibrils were performed as previously reported (Yoshikawa et al., 1999). A culture dish, in which myofibril preparations were attached to cover slip as above, was set on the stage of the optical microscope. Appropriate single myofibrils firmly attached to cover slip were selected and scanned by the tip of AFM cantilever to capture AFM images. On the other hand, the transverse stiffness of myofibrils was estimated based on forcedistance curves which were obtained by having the tip of AFM cantilever vertically approach appropriate loci of myofibril preparations. The force applied to the cantilever was calculated based on the deflection of cantilever and its spring constant. The stiffness was determined as the indentation of preparations vs. the force ap- plied to the cantilever. So as to minimally deform the myofilament lattice structures of myofibrils (Squire, 1981), the data points of force-distance curves at 20-30 $\mathrm{nm}$ indentations of preparations were used for the determination of the transverse stiffness. Further as mechanical compression by the cantilever tip could irreversibly damage surface structures of myofibril preparations, the very first force-distance curve obtained at each fresh locus was used for analysis.

Measurements were made at room temperature $\left(22-25^{\circ} \mathrm{C}\right)$.

\section{Chemicals}

CANP and ATP were purchased from Sigma Chemicals Co. (St. Louis, MO, USA). Other chemicals were of analytical grade and purchased from Wako Chemical Co. (Osaka, Japan).

\section{Results and Discussion}

\section{AFM imaging}

Fig. 1(A) shows a typical phase-contrasted video image of an intact single myofibril in rigor state attached to cover slip and observed under optical microscope. Single myofibril preparations were ca. $1 \mu \mathrm{m}$ in diameter and had characteristic periodical sarcomere structures with A-, I-, Z-, and Hbands (Bagshaw, 1993). Their sarcomere spacing was ca. $2.2 \mu \mathrm{m}$, indicating that the thin and thick filaments were fully overlapped (Yuri et al., 1998).

An AFM cantilever was positioned and scanned over single myofibril preparations to capture their AFM images. AFM images of single myofibrils thus obtained were ca. 1.0 $\mu \mathrm{m}$ in diameter having periodical structures at ca. $2.2 \mu \mathrm{m}$ intervals as can typically be seen in Fig. 2(A). Each unit

\section{(A)}
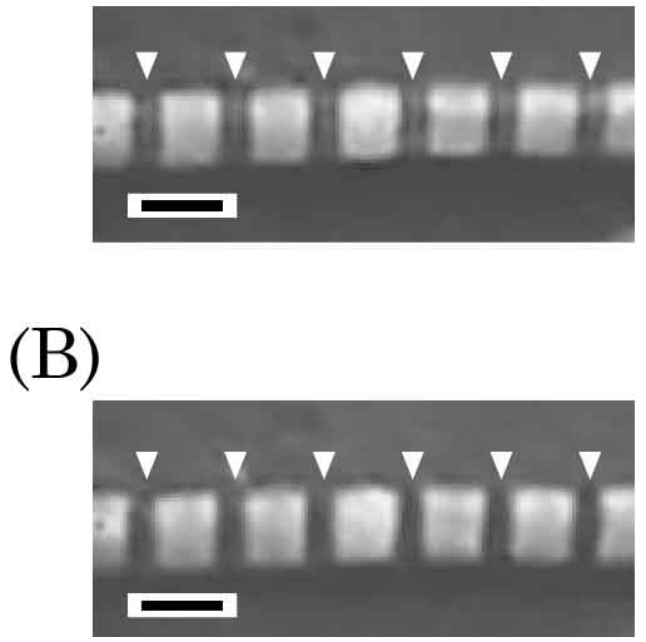

Fig. 1. Phase-contrasted video images of single myofibrils. (A) An intact myofibril and (B) a CANP-treated myofibril. Arrows indicate locations of the Z-band. Scale bars, $2 \mu \mathrm{m}$. 
structure was composed of broad filamentous bands, ca. 1.2 and ca. $0.5 \mu \mathrm{m}$ in width, interspaced with narrow bands of ca. 100-200 nm in width. At the middle of the broad band could be seen a faint band of ca. $0.15 \mu \mathrm{m}$ in width. These AFM images were essentially the same as reported previously (Yoshikawa et al., 1999), and very similar to the sarcomere structures observed under optical microscope.

Fig. 1(B) shows a typical phase-contrasted video image of a CANP-treated single myofibril in rigor state attached to cover slip and observed under optical microscope. By comparing the images of CANP-treated myofibrils with those of intact myofibrils, it was clearly noted that both of the myofibril preparations had essentially the same structures, except that Z-bands were absent from CANP-treated preparations as reported by Reddy et al. (1975).

As in the cases of intact myofibrils, AFM images of CANP-treated myofibril preparations were captured. While fibrous structures having periodical bands were expected from the optical microscopic images, such AFM images could not be obtained under the conditions employed for capturing images of intact myofibril preparations. Instead, careful and extensive trials only captured heavily disturbed fibrous images as can typically be seen in Fig. 2(B). This strongly suggests that the surface structures of CANP-treated myofibrils were disrupted mechanically by scanning with the cantilever tip to capture images. Considering that Z-bands were selectively removed in CANP-treated myofibrils, we conclude that the Z-band is an essential component to maintain the sarcomere structure of myofibrils intact against external mechanical stress.

\section{Transverse stiffness distribution}

Transverse stiffness distributions of intact single myofibrils

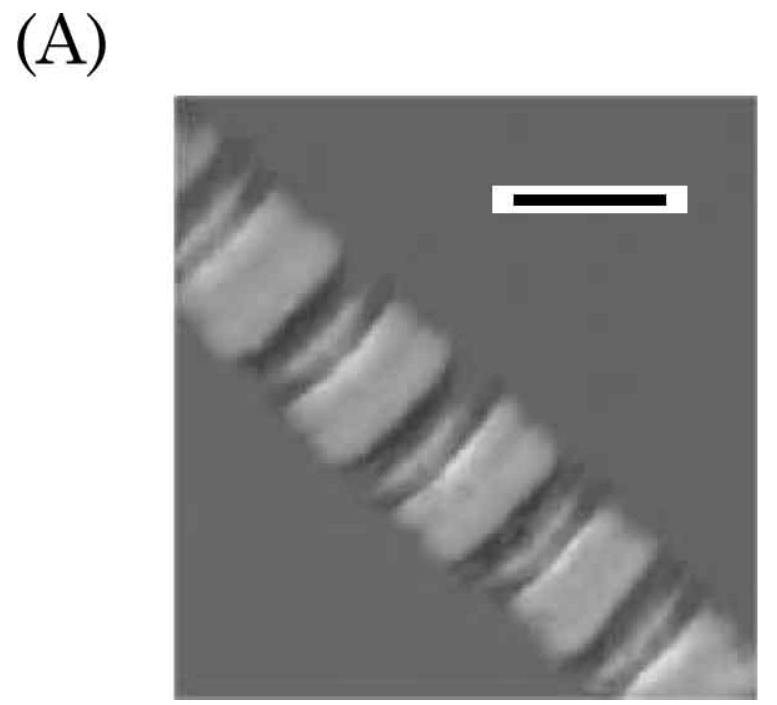

were determined based on a series of force-distance curves obtained at various loci along preparations. Thus, starting from an appropriate locus, the position of the tip of AFM cantilever was shifted stepwise at $50-100 \mathrm{~nm}$ intervals along myofibril preparations, and a force-distance curve obtained at each locus. Fig. 3(A) shows typical force-distance curves for a narrow rigid band and a locus in between narrow rigid bands of an intact single myofibril preparation, the transverse stiffness for which was determined to be 11.1 , and $3.2 \mathrm{pN} / \mathrm{nm}$, respectively. Based on a series of force-distance curves thus obtained, the distributions of the transverse stiffness of myofibrils were determined. The obtained result is shown in Fig. 3(B). It can be seen that periodical rigid bands having a width of ca. $100 \mathrm{~nm}$ were located at ca. $2.2 \mu \mathrm{m}$ intervals while all the other loci were roughly two- to three-fold less rigid than the narrow rigid bands. By carefully measuring force-distance curves, the width of the narrow rigid band was estimated to be 50-100 nm.

Similarly transverse stiffness distributions of CANPtreated myofibrils were examined. A typical force-distance curve obtained for a CANP-treated single myofibril is shown in Fig. 3(A), the transverse stiffness for which was determined to be $2.1 \mathrm{pN} / \mathrm{nm}$. Very similar force-distance curves were obtained all along CANP-treated preparations, which were comparable to those obtained at loci in between narrow rigid bands of intact preparations. This strongly suggests that force-distance curves of CANP-treated myofibril preparations were successfully obtained under the conditions employed for intact myofibril preparations. Considering that clear AFM images could not be obtained for CANP-treated myofibril preparations as mentioned above, this suggests that the surface structures of preparations were

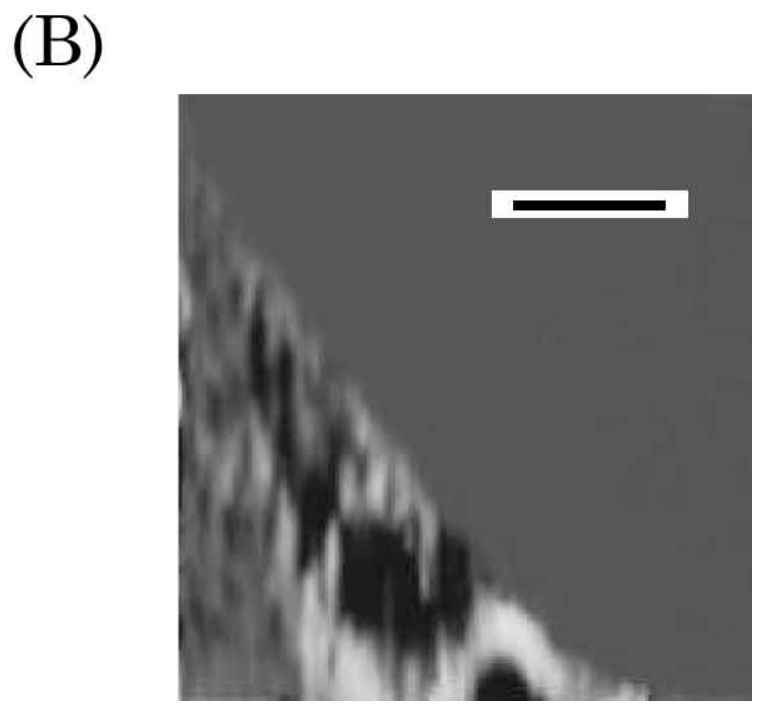

Fig. 2. (A) A typical AFM image of an intact single myofibril in rigor state. Scale bar, $2 \mu \mathrm{m}$. (B) A typical AFM image of a CANP-treated single myofibril in rigor state. Scale bar, $2 \mu \mathrm{m}$. 
(A)

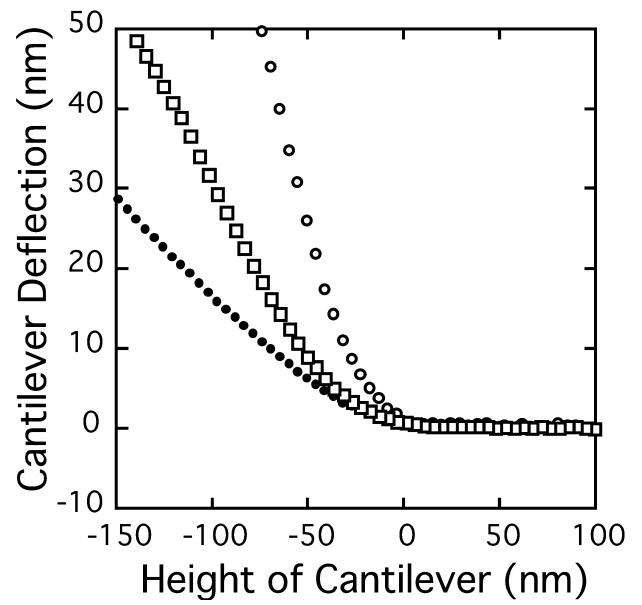

(B)

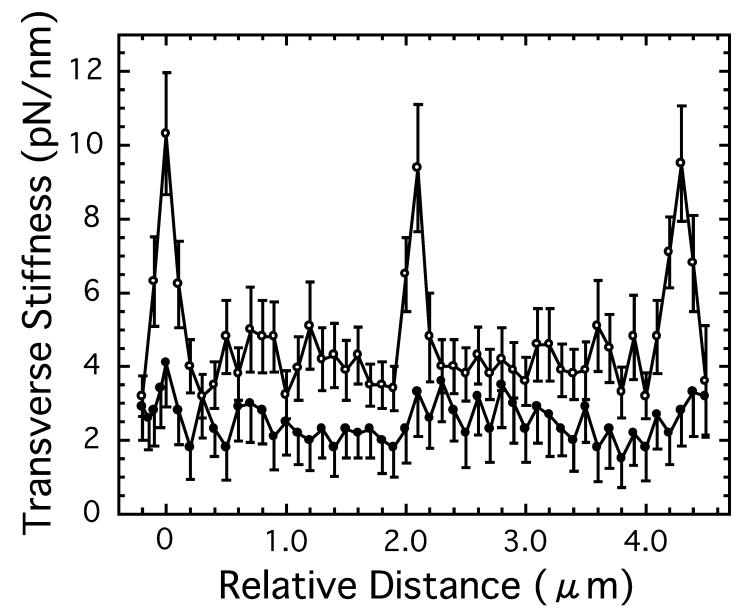

Fig. 3. (A) Typical force-distance curves for intact myofibril $(\bigcirc)$ at the rigid narrow band and $(\square)$ at the middle position between narrow rigid bands, and for $(\bigcirc)$ CANP-treated myofibril. (B) Transverse stiffness distributions of myofibrils in rigor state. $(\bigcirc)$ Intact myofibril $(\mathrm{n}=30)$ and $(\mathbf{O})$ CANP-treated myofibril $(\mathrm{n}=30)$. The bar represents the S.E.M. In each series of data obtained the most rigid locus was shifted horizontally to the origin of the abscissa.

not damaged significantly by force-distance measurements where the tip of cantilever transiently pressed against the preparations once at each locus, while they were heavily damaged by AFM imagings where the tip of cantilever continuously scanned the preparations. Thus a series of forcedistance curves was obtained along CANP-treated myofibrils as in the case of intact preparations, and the corresponding stiffness distributions determined. The obtained result is shown in Fig. 3(B). It can be clearly seen that narrow rigid bands present in intact myofibril preparations were absent in CANP-treated preparations; i.e., the stiffness was almost the same all along the preparations, 1.8 to 4.1 $\mathrm{pN} / \mathrm{nm}$, which was four- to five-fold less rigid than that for the narrow rigid bands of intact myofibrils, ca. $10 \mathrm{pN} / \mathrm{nm}$.

As can be seen in Fig. 3(B), vague band structures having slightly more rigidity than other loci were observed at ca. 2 $\mu \mathrm{m}$ intervals in CANP-treated preparations. These may be traces of Z-bands not completely removed by the CANPtreatment. Further all the loci examined in CANP-treated myofibrils were less rigid compared with various loci in intact preparations, suggesting that the overall structure of the myofilament lattice of myofibrils became fragile without the Z-bands.

As Z-bands were selectively removed from CANP-treated myofibril preparations, the above results clearly indicate that the rigid band structures of ca. $100 \mathrm{~nm}$ in width present in between broad bands in the AFM image of intact myofibril shown in Fig. 2(A) are the Z-bands. The width of the rigid band is consistent with that for the Z-bands observed in electron micrographs of skeletal muscle, $67 \mathrm{~nm}$ (Millevoi et al., 1998; Squire, 1981).

It is generally believed that the M-line is a rigid component, like the Z-band, supporting the sarcomere structure of muscle (Squire, 1981). Our AFM studies showed, however, that the transverse stiffness at the middle of adjacent Zbands, where the M-line should be located (Bagshaw, 1993), is two to three times less rigid than that for the Zband as can typically be seen in Fig. 3(B). Further it has been reported that (a) Z-bands remained intact even after thick filaments were selectively extracted from muscle fibers (Huxley, 1972); that (b) when rigor muscle fibers were overstretched, M-lines completely disappeared while Z-bands became skewed but remained almost intact (Suzuki and Sugi, 1983); and that (c), associated with extensive contractions, myofibril preparations developed damages in the sarcomere structures initiating near the A-bands, not at the Z-bands (Yuri et al., 1998). These results strongly suggest that the Z-band is the most rigid component in the sarcomere structure, and that the M-line is not so rigid as the Zband. In any case we concluded that the Z-band is a rigid disc that plays the primary role in supporting the sarcomere structure of skeletal muscle.

Acknowledgments. The authors would like to thank Dr. Y. Okamoto, Department of Biotechnology, Muroran Institute of Technology, for his helpful suggestions. This work was supported in part by Grant-in-Aids for Scientific Research (C-09680658 and C-12680660) and a Special Grant for Promotion of Science (12030223) from the Ministry of Education, Science, Sports, and Culture of Japan to T.Y.

\section{References}

Bagshaw, C.R. 1993. Muscle Contraction. Chapman \& Hall, London, pp. 21-24.

Engel, A. and Gaub, H. 1998. Biological systems. In Procedures in Scanning Probe Microscopies (R.J. Colton et al., eds.). John-Wiley \& Sons, New York, pp. 385-505.

Huxley, H.E. 1972. Molecular basis of contraction in cross-striated muscles. In The Structure and Function of Muscle (G.H. Bourne, ed.). Academic Press, San Diego, Vol. 1, pp. 301-387. 
Millevoi, S., Trombitas, K., Kolmerer, B., Kostin, S., Schaper, J., Pelin, K., Granzier, H., and Labeit, S. 1998. Characterization of nebulette and nebulin and emerging concepts of their roles for vertebrate Z-discs. $J$. Mol. Biol., 282: 111-123.

Reddy, M.K., Etlinger, J.D., Rabinowitz, M., Fischman, D.A., and Zak, R. 1975. Removal of Z-lines and $\alpha$-actinin from isolated myofibrils by a calcium-activated neutral protease. J. Biol. Chem., 250: 4278-4284.

Squire, J. 1981. The Structural Basis of Muscular Contraction. Plenum Press, New York, pp. 265-380.

Suzuki, S. and Sugi, H. 1983. Extensibility of the myofilaments in vertebrate skeletal muscle as revealed by stretching rigor muscle fibers. $J$.
Gen. Physiol., 81: 531-546.

Yoshikawa, Y., Yasuike, T., Yagi, A., and Yamada, T. 1999. Transverse elasticity of myofibrils of rabbit skeletal muscle studied by atomic force microscopy. Biochem. Biophys. Res. Comm., 256: 13-19.

Yuri, K., Wakayama, J., and Yamada, T. 1998. Isometric contractile properties of single myofibrils of rabbit skeletal muscle. J. Biochem., 124: $565-571$.

(Received for publication, December 7, 2000

and in revised form, January 4, 2001) 